\title{
Awake-craniotomy for cavernoma resection
}

\author{
Eva Pamias-Portalatin, MD, ${ }^{1}$ Ivan Segura Duran, MD, ${ }^{1}$ James Ebot, DO, ${ }^{1}$ Elird Bojaxhi, MD, ${ }^{2}$ \\ William Tatum, DO, ${ }^{3}$ and Alfredo Quiñones-Hinojosa, MD ${ }^{1}$
}

Departments of ${ }^{1}$ Neurosurgery, ${ }^{2}$ Anesthesiology and Perioperative Medicine, and ${ }^{3}$ Neurology, Mayo Clinic Hospital, Jacksonville, Florida

Cavernomas make up approximately $8 \%-15 \%$ of all intracranial vascular malformations, and the most common presenting symptom is seizures. Complete resection of the cavernoma and removal of the surrounding gliotic core presents a cure but poses a challenge if an eloquent brain is involved or with incomplete resection of the epileptogenic foci. The authors present the case of a 53-year-old man with intractable seizures from a left posterior temporal lobe cavernoma who underwent an awake craniotomy with intraoperative seizure monitoring via electrocorticography.

The video can be found here: https://youtu.be/vxaikozg2g4.

KEYWORDS cavernoma; awake craniotomy; seizures; video 